\title{
COMBINATORIAL STRUCTURES AND GROUP INVARIANT PARTITIONS ${ }^{1}$
}

\author{
DENNIS E. WHITE AND S. G. WILLIAMSON ${ }^{2}$
}

\begin{abstract}
ABSTRACr. If a group acts on a set, an action of the group is induced on the partitions of the set. A formula is developed for the number of partitions invariant under this action. The formula is extended to count combinatorial objects such as labeled rooted trees or permutations defined on the invariant partitions.
\end{abstract}

1. Introduction. Let $G$ be a finite group acting on a finite set $S$. This action induces a natural action on the partitions $\Pi(S)$ of $S$. A partition $\pi \in \Pi(S)$ is $G$-invariant if the stabilizer subgroup $G_{\pi}=G$. $G$-invariant partitions arise naturally in the study of isomorph free backtrack algorithms [2].

The purpose of this paper is to discuss the construction and enumeration of combinatorial structures on $G$-invariant partitions. In a very interesting dissertation [3], M. Klass has characterized all $G$-invariant partitions and given formulas for computing the number of such partitions. Klass's results are expressed in probabilistic terms and involve expectations of products of dependent random variables, themselves sums of independent Poisson random variables. Computationally Klass's results involve the construction of marks for the group $G$ [1], [4] plus additional identities related to Stirling numbers of the second kind. Our analogous result relies on the marks of $G$ and is derived and stated directly in combinatorial terms (Theorem 1, below). In Theorem 2 this result is extended to include the enumeration of structures (rooted trees, graphs, permutation cycles, partitions with restrictions on block size, etc.) on $G$-invariant partitions. Applications and computational algorithms based on these results will be discussed in detail in a subsequent paper.

2. Principal results. Let $H, K$ be subgroups of $G$. We define

$$
M_{H}(K)=\frac{1}{|H|} \sum_{\sigma \in G} \chi\left(\sigma K \sigma^{-1} \subset H\right)
$$

where

Received by the editors July 15, 1973 and, in revised form, January 10, 1975.

AMS (MOS) subject classifications (1970). Primary 05A17, 05A19; Secondary 05C05, $20 \mathrm{~B} 99$.

Key words and phrases. Partitions, group-invariant partitions, group actions, marks of a group, labeled rooted trees, permutations.

1 Research sponsored by the Air Force Office of Scientific Research, Air Force Systems command, USAF, under AFOSR Contract/Grant No. 71-2089. The United States Government is authorized to reproduce and distribute reprints for Government purposes notwithstanding any copyright notation hereon.

2 The authors wish to thank their colleague, Professor Adriano M. Garsia, for suggesting this problem. 


$$
\chi(\text { statement })= \begin{cases}1 & \text { if statement is true } \\ 0 & \text { if statement is false. }\end{cases}
$$

$M_{H}(K)$ is called the mark of $H$ at $K$ and is discussed in [1], [4]. It is easily shown that

$$
M_{H}(K)=\frac{|G|[H \supset K]}{|H|[H]}=\frac{|N(H)|}{|H|}[H \supset K],
$$

where $|G|$ denotes the cardinality of $G,[H]$ denotes the number of subgroups of $G$ conjugate to $H,[H \supset K$ ] denotes the number of subgroups of $G$ containing $K$ and conjugate to $H$, and $N(H)$ is the normalizer of $H$ in $G$.

Let $\Delta$ be a system of orbit representatives for the action of $G$ on $S$. Let $\Pi(\Delta)$ be the partitions of $\Delta$. Let $\mathcal{H}$ be a complete set of nonconjugate subgroups of $G$. Let $G_{t}$ be the stabilizer subgroup of $t \in S$.

THEOREM 1. The number of $G$-invariant partitions of $S$ is given by

$$
\sum_{\delta \in \Pi(\Delta)} \prod_{A \in \delta} \sum_{H \in \mathcal{K}} \frac{1}{M_{H}(H)} \prod_{t \in A} M_{H}\left(G_{t}\right) .
$$

As we note in $\S 3$, this follows from Theorem 2 below.

Consider a family $\widetilde{\mathscr{Y}}$ of finite sets $\left\{X_{Q}: Q \subset S\right\}$ satisfying $X_{Q} \cap X_{Q^{\prime}}=\varnothing$ if $Q \neq Q^{\prime}$ and $\left|X_{Q}\right|=\left|X_{Q^{\prime}}\right|$ if $|Q|=\left|Q^{\prime}\right|$. For example:

(1) $X_{Q}$ could denote all labeled rooted trees on $Q$.

(2) $X_{Q}$ could denote all cyclic permutations of $Q$.

(3) $X_{Q}$ could denote $\{Q\}$ if $|Q| \leqslant m$, or $\varnothing$ if $|Q|>m$.

For such a family $\mathscr{F}$ and a given partition $\pi \in \Pi(S)$ we define the set of $\mathcal{F}_{\mathcal{F}}$-structures on $\pi$ to be $\times_{B \in \pi} X_{B}$. If $\pi=\left\{B_{1}, \ldots, B_{k}\right\}$ and if $X_{B_{i}}$ consists of $b_{i}$ elements, then the set of $\mathscr{F}$-structures on $\pi$ consists of $b_{1} b_{2} \cdots b_{k}$ objects, each of the form $\left\{x_{B_{1}}, x_{B_{2}}, \ldots, x_{B_{k}}\right\}$ where $x_{B_{i}} \in X_{B_{i}}$. Observe that if $\pi \neq \pi^{\prime}$ then the $\widetilde{y}$-structures on $\pi$ and the $\widetilde{F}$-structures on $\pi^{\prime}$ are disjoint sets. In example (1), above, the set of $\widetilde{F}$-structures is the forest of rooted trees on $\pi$. In example (2) the set of $\mathscr{f}$-structures is the set of all permutations whose cycles are constructed from the blocks of $\pi$. In example (3) the set of $\widetilde{F}$-structures on $\pi$ is the set $\left\{\left(B_{1}, B_{2}, \ldots, B_{p}\right)\right\}$ if $\pi=\left\{B_{1}, B_{2}, \ldots, B_{p}\right\}$ and $\left|B_{i}\right| \leqslant m$ for all $i$. Otherwise the set of $\widetilde{\gamma}$-structures on $\pi$ is empty.

Let $\mathcal{C} \subset \Pi(S)$. By the set of $\mathcal{F}$-structures on $\mathcal{E}$ we shall mean the union over all $\pi \in \mathcal{C}$ of the set of $\widetilde{F}$-structures on $\pi$. This union is disjoint by the above remarks.

THEOREM 2. The number of $\widetilde{F}$-structures on the G-invariant partitions is

$$
\sum_{\delta \in \Pi(\Delta)} \prod_{A \in \delta} \sum_{H \in \mathcal{H}} \frac{1}{M_{H}(H)}\left[f\left(|H| \sum_{t \in A} \frac{1}{\left|G_{t}\right|}\right)\right]^{|G| /|H|} \prod_{t \in A} M_{H}\left(G_{t}\right),
$$

where $f(k)=\left|X_{B}\right|$ for any $B \subset S,|B|=k$.

Note that in example (1) $\left|X_{Q}\right|=|Q|^{|Q|}-1$, in example (2) $\left|X_{Q}\right|=$ $(|Q|-1)$ !, and in example (3) 


$$
\left|X_{Q}\right|= \begin{cases}1 & \text { if }|Q| \leqslant m, \\ 0 & \text { if }|Q|>m .\end{cases}
$$

3. Proofs. By example (3) with $m=|S|$, Theorem 2 implies Theorem 1. We first consider the case where $G: S$ is transitive. The general case will be deduced by piecing together the $G$-invariant partitions of the orbits of $G: S$ ( $G: S$ means "the action of $G$ on $S$ ").

If $G: S$ is transitive and $\pi$ is $G$-invariant, then the action of $G$ on the blocks of $\pi(G: \pi)$ is transitive. Given any $B \subset S$, if for any $g \in G$ either $g B=B$ or $g B \cap B=\varnothing$, then $\pi=\{g B: g \in G\}$ is a $G$-invariant partition of $S$. Clearly all $G$-transitive, $G$-invariant partitions arise in this manner. Since $G: S$ is transitive, we identify $S$ with the set $G / G_{s}$ where $s \in S$. The action $G: G / G_{s}$ is the standard action of $G$ on the cosets $\left\{g G_{s}: g \in G\right\}$. Let $K=G_{s}$.

Lemma 3 (Klass [3]). If $G: S$ is transitive, there is a one-to-one correspondence between $G$-invariant partitions of $S$ and subgroups $H$ of $G$ such that $H \supset K$. For such an $H$ the associated $G$-invariant partition is generated by the block $B=\{h s: h \in H\}$.

Proof. We work with the equivalent action of $G$ on $G / K$, identifying $s$ with the coset $K$. Let $B=\{h K: h \in H\}=H / K$. For $g \in G$ either $g B=B$ or $g B \cap B=\varnothing$. Thus for each $H \supset K, H / K$ generates a $G$-invariant partition. If $H \neq H^{\prime} \supset K$, then $H^{\prime} / K \neq H / K$ so each $H \supset K$ generates a distinct partition.

We now show that all $G$-invariant partitions arise in this manner. If $\pi$ is $G$-invariant, let $B$ be the block of $\pi$ containing $K$. Let $H=G_{B}$. For $h \in H$, $h B=B$ so $h K \in B$ and thus $\{h K: h \in H\} \subseteq B$. On the other hand, if $g K \in B$, then $g B \cap B \neq 0$. Since both $B$ and $g B$ are blocks of a partition, $g B=B$. Thus $K \subseteq H$ and $B=\{h K: h \in H\}=H / K$.

REMARK 4. Note that $G_{\sigma A}=\sigma G_{A} \sigma^{-1}$ for any subset $A \subseteq S$. When $G$ is transitive, the set of stabilizers of the blocks of a $G$-invariant partition $\pi$ is an entire conjugacy class of subgroups of $G$. Therefore, by Lemma 3, there must be exactly $[H \supset K] G$-invariant partitions $\pi$ such that $G_{B}=H$ for some block $B \in \pi$.

REMARK 5. For any transitive action $G: Q$, the number (when nonzero) of elements $p \in Q$ such that $G_{p}=H$ is $|N(H)| /|H|$. If $\pi$ is $G$-invariant, let $Q=\pi$. Then the number of blocks $B$ of $\pi$ such that $G_{B}=H$ is $|N(H)| /|H|$ $=M_{H}(H)$.

REMARK 6. Combining Remarks 4 and 5 we see that the number of subsets $B \subset S=G / K, G_{B}=H$, which generate $G$-invariant partitions is $M_{H}(K)$. This remark gives an interesting combinatorial interpretation of the marks of $G$.

We now consider the general case where the $G: S$ may be intransitive. Observe that the restriction of a $G$-invariant partition $\pi$ to an orbit of $G: S$ is itself a $G$-invariant partition. Let $\left\{O_{t}: t \in \Delta\right\}$ denote the orbits of $G: S$ and let $\left\{\pi_{t}: t \in \Delta\right\}$ denote the restrictions of $\pi$ to these orbits. Let $B \in \pi$ and let $\left\{B_{t}: t \in \Delta\right\}$ be the restrictions of $B$ to the orbits. Then one can easily show that $H=G_{B}=G_{B_{i}}$ for all $i$ such that $B_{i} \neq \varnothing$. For any block $B^{\prime} \in \pi$ which is 
equivalent to $B$ under $G: \pi$ we have that $\left\{i: B_{i}^{\prime} \neq \varnothing\right\}=\left\{i: B_{i} \neq \varnothing\right\}$. If $B^{\prime}$ is not equivalent to $B$, then $\left\{i: B_{i}^{\prime} \neq \varnothing\right\}$ and $\left\{i: B_{i} \neq \varnothing\right\}$ are disjoint. Thus the orbits of $G: \pi$ define a partition $\delta \in \Pi(\Delta)$. Associated with each block $A$ of $\delta$ is a conjugacy class of subgroups of $G$. This class is associated with the stability subgroups of the orbit of $\pi$ which defines $A$ as described above.

It remains only to enumerate the $G$-invariant partitions associated with a given partition $\delta \in \Pi(\Delta)$ and a given assignment $\nu \in \mathcal{H}^{\delta}$ of conjugacy classes to the blocks of $\delta$. Let $A$ be a block of $\delta$ and let $H=\nu(A)$. Let $\mathscr{G}_{A, H}$ denote the set of $G$-transitive, $G$-invariant partitions of $\cup_{t \in A} O_{t}$ whose stabilizer subgroup class is that of $H$. Let $b_{A, H}=\left|\mathscr{乃}_{A, H}\right|$. Then the number of $G$-invariant partitions of $S$ is

$$
\sum_{\delta \in \Pi(\Delta)} \sum_{\nu \in \mathcal{H}^{\delta}} \prod_{A \in \delta} b_{A, \nu(A)}=\sum_{\delta \in \Pi(\Delta)} \prod_{A \in \delta} \sum_{H \in \mathscr{K}} b_{A, H}
$$

At this point we observe that if $\pi$ is any $G$-invariant partition whose orbits define $\delta \in \Pi(\Delta)$, then the size of the block $B \in \pi$ associated with the block $A \in \delta$ and the subgroup $H \in \mathcal{K}$ is $|H| \sum_{t \in A} 1 /\left|G_{t}\right|$. This follows since $\left|B_{t}\right|=\left|G_{B_{t}}\right| /\left|G_{t}\right|=|H| /\left|G_{t}\right|$ for each $t \in A$.

We now compute the numbers $b_{A, H}$. Note that the number of ways of choosing one subset $Q_{t}$ from each orbit $O_{t}, t \in A$, such that $G_{Q_{t}}=H$ is $\prod_{t \in A} M_{H}\left(G_{t}\right)$ by Remark 6. However, any two such choices $\left\{Q_{t}: t \in A\right\},\left\{Q_{t}^{\prime}\right.$ : $t \in A\}$ define the same partition $\theta$ of $\cup_{t \in A} O_{t}$ if and only if for some $g \in G$, $g Q_{t}=Q_{t}^{\prime}$ for all $t \in A$. However, since $G$ acts transitively on $\theta$ the number of such choices $\left\{Q_{t}^{\prime}: t \in A\right\}$ for any choice $\left\{Q_{t}: t \in A\right\}$ is $|N(H)| /|H|$ by Remark 5. Thus $b_{A, H}=\left(M_{H}(H)\right)^{-1} \Pi_{t \in A} M_{H}\left(G_{t}\right)$ and by the remark following equation (2), the number of $\mathscr{F}^{\prime}$-structures on any element of $\mathscr{R}_{A, H}$ is

$$
k_{A, H}=\left[f\left(|H| \sum_{t \in A} \frac{1}{\left|G_{t}\right|}\right)\right]^{|G| /|H|},
$$

since there are $|G| /|H|$ blocks in any element of $\bigcap_{A, H}$. Thus by (2) the number of $\mathscr{F}$-structures on $S$ is

$$
\sum_{\delta \in \Pi(\Delta)} \prod_{A \in \delta} \sum_{H \in \mathscr{K}} k_{A, H} b_{A, H}
$$

This completes the proof of Theorem 2 and consequently Theorem 1 .

\section{REFERENCES}

1. W. Burnside, Theory of groups of finite order, 2nd ed., Dover, New York, 1955. MR 16, 1086.

2. J. P. Fillmore and S. G. Williamson, On backtracking: a combinatorial description of the algorithm, SIAM J. Comput. (1) 3 (1974), 42-55.

3. M. J. Klass, Enumeration of partition classes induced by permutation groups, Ph.D. Dissertation, Department of Mathematics, UCLA, 1972.

4. D. E. White, Classifying patterns by automorphism group: an operator theoretic approach, Discrete Math. (to appear).

School of Mathematics, University of Minnesota, Minneapolis, Minnesota 55455

Department of Mathematics, University of California, San Diego, la Jolla, CALIFORNIA 92037 